\title{
Mechanism of antrocytic connexin-43 autophagy degradation in oxygen-glucose deprivation and its effect on neuroinflammation
}

\author{
Xinyu Wang \\ jilin \\ Liangshu Feng \\ jilin
}

Meiying Xin

jilin

Yulei Hao

jilin

Xu Wang

jilin

Pei Shang

jilin

Mingming Zhao

jilin

Shuai Hou

jilin

Yunhai Zhang

suzhou

Yun Xiao

suzhou

Di Ma

jilin

Jiachun Feng ( $\square$ fengjcfrank2017@163.com )

\section{Research}

Keywords: Oxygen-glucose deprivation, Astrocytes, Cx43, OPTN, NDP52, PINK1, Autophagy, Phosphorylation

Posted Date: December 13th, 2019

DOI: https://doi.org/10.21203/rs.2.18795/v1 
License: (c) (i) This work is licensed under a Creative Commons Attribution 4.0 International License. Read Full License 


\section{Abstract}

Background : Connexin $43(\mathrm{Cx} 43)$ are the most widely distributed gap junction proteins in the nervous system. Cx43 enables cell-to-cell communication and plays an important role in ion transport, substrate exchange and delivery of information, which have been implicated in cerebral ischemia injury. Our previous work revealed the relationships between $\mathrm{Cx} 43$ and glia-mediated neuroinflammation through the release of ATP in oxygen-glucose deprivation (OGD), which means degradation of $\mathrm{Cx} 43$ may improve neuroinflammatory damage during OGD injury. However, the roles of Cx43 degradation and neuroinflammation caused by OGD remain unclear.

Methods: We used primary cultured astrocytes treated with OGD as an in vitro model of cerebral ischemia injury and we used middle cerebral artery occlusion (MCAO) model as an in vivo model of cerebral ischemia. HeLa cells were used in overexpression experiments. Cx43 protein levels were determined by western blotting. The interaction between $\mathrm{Cx} 43$ and related autophagy receptors was determined by coimmunoprecipitation and immunofluorescence. The gene knockdown (KD) of ATG5, OPTN, NDP52, PINK1 and Cx43 was applied by siRNA transfection. Related cytokines were detected by cytometric bead assay.

Results: We found that Cx43 protein levels increased after ischemia in gene KD of ATG5, OPTN, NDP52 and PINK1 primary astrocytes. The interaction of Cx43 with OPTN, NDP52 and PINK1 was increased after cerebral ischemia injury in vitro and vivo. While the interaction was weakened after point mutation of Cx43 at Ser368, Tyr265 and Tyr247. Meanwhile, IL-10 upregulated during OGD after KD of ATG5, OPTN, NDP52 and PINK1 in astrocytes, while TNF downregulated during OGD after KD of ATG5, OPTN, NDP52 and PINK1 in astrocytes.

Conclusions: Our results suggest that degradation of $\mathrm{Cx} 43$ is caused by selective autophagy during ischemia injury and the autophagy degradation of $\mathrm{Cx} 43$ plays important roles in neuroinflammation mediated by OGD injury. Treatment targeting $\mathrm{Cx} 43$ degradation pathway can improve neuroinflammation responses induced by OGD injury, which provide novel therapeutic strategies and crosstalk between autophagy and neuroinflammation.

\section{Introduction}

Ischemic stroke is a major cause of death and disability in humans worldwide. Ischemia strokes seriously endanger the health and lives of middle-aged and older people, in particular.

Cx43, a gap junction protein, is widely present in the nervous system and is an important component of intercellular communication machinery ${ }^{[1,2]}$. Following ischemia, the contents, modification, and distribution of $\mathrm{Cx} 43$ are significantly altered, affecting the outcome after ischemia. For example, in an anoxic cardiomyocyte model, the expression levels of $\mathrm{Cx} 43$ and p-Cx43 change over time ${ }^{[3]}$. In hypoxic injury cases, within 15 minutes, no significant change in $\mathrm{Cx} 43$ expression was noted ${ }^{[4,5]}$. After several 
hours, the expression level of $\mathrm{Cx} 43$ had significantly reduced ${ }^{[6]}$. This change first appeared as its internalization ${ }^{[7]}$, and then, the total amount of $\mathrm{C} \times 43$ decreased $^{[4]}$. All these works proved that $\mathrm{C} \times 43$ is degraded during ischemia injury. Moreover, gap junction is degraded by autophagy under normal conditions $^{[8]}$. However, the role of $\mathrm{Cx} 43$ degradation remain unknown during cerebral ischemia injury.

Autophagy, one of the pathways of protein degradation, is the clearing process of cytoplasmic materials by lysosomes. Interestingly, autophagy is selective ${ }^{[9]}$. The development of selective autophagy requires autophagy receptors. Of these, OPTN and NDP52 are the autophagy receptors of mitophagy ${ }^{[10]}$. They directly interact with LC3 (microtubule-associated protein light chain 3) on the isolation membrane through the LC3-interacting region. Subsequently, the complex is incorporated into the autophagosome and is then degraded. Mitophagy happens under ischemia stress, at the same time, Cx43 can be found in the mitochondria ${ }^{[11]}$. Thus, OPTN and NDP52 may be the autophagy receptors for $\mathrm{Cx} 43$ degradation during ischemia injury.

Posttranslational modification (PTM) of protein is needed during its degradation. Phosphorylation and ubiquitination are the most common. It is now cleared that $\mathrm{Cx} 43$ is phosphorylated at Ser368 by PKC and at Tyr247, Tyr265 by Src ${ }^{[12]}$. PKC and Src kinases are activated during global ischemia ${ }^{[13-16]}$, and the phosphorylation of $\mathrm{C} \times 43$ can mediate its degradation ${ }^{[17]}$. Many studies have confirmed that $\mathrm{C} \times 43$ is also modified by ubiquitin ${ }^{[18-20]}$. Meanwhile there is evidence that ubiquitin is strongly linked to $\mathrm{Cx} 43$ after the activation of $\mathrm{PKC}^{[21]}$. Interestingly, some studies have confirmed that ubiquitin kinase PINK1 phosphorylates ubiquitin at Ser65 to activate mitochondrial degradation during mitophagy ${ }^{[22,23]}$. This means $\mathrm{pUb}(\mathrm{S} 65)$ can be a signal for protein degradation caused by autophagy. All above research reminds us that $\mathrm{Cx} 43$ degradation required its phosphorylation and ubiquitination.

Besides, our previous work suggested that treatment targeting microglia Cx43 like carbenoxolone (CBX) and Gap 26 can improve neuroinflammation mediated by cerebral ischemia injury ${ }^{[24,25]}$. This suggests that the degradation of Cx43 may play critical roles in cerebral ischemia injury and related neuroinflammation.

Here, we examine the roles of $\mathrm{Cx} 43$ degradation and neuroinflammation caused by cerebral ischemia. We report that Cx43 is phosphorylated at Ser368 by PKC, and at Tyr247 and Tyr265 by Src kinases after OGD injury. After that, pUb(S65) combines with phosphorylated Cx43. Then, autophagy receptor NDP52 and OPTN recognize modified $\mathrm{Cx} 43$ according to its ubiquitin-binding domains (UBDs), and they directly interact with LC3 on the isolation membrane through the LC3-interacting region. Subsequently, those protein complexes are incorporated into the autophagosome and are then degraded. Besides, the autophagy degradation of Cx43 plays important roles in neuroinflammation mediated by OGD injury. Treatment targeting $\mathrm{Cx} 43$ degradation pathway can affect neuroinflammation responses induced by OGD injury, bringing more applications to improve glia-mediated neuroinflammation.

\section{Materials And Methods}


Animals

All experiments were performed with either newborn or adult male C57BL/6 mice, weighing 20-25 g. All animal experiments were performed with the approval of the Institutional Animal Care and approved by the Animals Ethics Committee of Jilin University of China (10 February 2014, NO. 2014-277).

\section{Cell culture and reagents}

Astrocytes were obtained from the cerebral cortices of one-day-old C57BL/6 mice. The cells were cultured in DMEM (Gibco, 11995065) containing 10\% fetal bovine serum (Gibco, 10099141) and 1\% penicillin/streptomycin (Gibco, 15140122) for 10 days. HeLa (American Type Culture Collection) were cultured in DMEM (Gibco, 11995065) containing 10\% fetal bovine serum (Gibco, 10099141) and 1\% penicillin/streptomycin (Gibco, 15140122). All cells were tested for mycoplasma contamination bimonthly using the PlasmoTest kit (InvivoGen).

The following drugs were used: baf A1 (Selleck, S1413, 400nM, 6h).

\section{OGD injury to astrocytes}

Astrocytes were washed with PBS three times and cultured in DMEM (no glucose) (Gibco, 11966025). Cells were designed to grow in an incubator with a mixture of 95\% N2, and $5 \% \mathrm{CO} 2$ (hypoxia) inlet for $3 \mathrm{~h}$ or $6 \mathrm{~h}$.

\section{SiRNA, plasmids and antibodies}

We purchased short interference RNAs for mouse Atg5, PINK1, OPTN, NDP52 and negative siRNA from Gene Pharma (Suzhou, China). All siRNA sequences have been listed in Supplementary Table 2. pCDNA3 HA-Ub and pCDNA3 PINK1-Myc were obtained from Miaoling (Wuhan, China). RFP-OPTN, RFP-NDP52, GFP-Cx43, GFP-Cx43 ${ }^{368 \mathrm{~A}}$ and GFP-Cx43 ${ }^{247 \mathrm{~A}+265 \mathrm{~A}}$ plasmids were designed by Gene Pharma (Suzhou, China) and these are all pGCMV plasmids. All plasmids sequences have been listed in Supplementary Table 1.

Primary antibodies: Cx43 (Abcam, ab79010), Cx43 (Millipore, AB1728-25UG), p-Cx43(S368) (Cell Signaling Technology, 3511S), p-Cx43(T265) (Invitrogen, PA5-37584), Atg5 (Abcam, ab108327), Betaactin (Abcam, ab8226), OPTN (GeneTex, GTX132575), NDP52 (GeneTex, GTX115378), GFP(Abcam, ab6556), LC3B (Abcam, ab192890), PINK1(Novus Biologicals, BC100-494), phospho-Ubiquitin (Ser65) (Cell Signaling Technology, 62802S), HA-Tag (Cell Signaling Technology, 3724T), RFP (Abcam, ab62341) and Myc-Tag (Cell Signaling Technology, 2272S). Secondary antibodies: Goat anti-rabbit IgG (Cell Signaling Technology, 7074S), Goat anti-mouse IgG (Cell Signaling Technology, 7076S), Mouse antirabbit IgG (Conformation Specific) (L27A9) (Cell Signaling Technology, 5127S), Goat anti-mouse IgG $(\mathrm{H}+\mathrm{L})$ (Alexa Fluor 488) (Abcam, ab150113) and Goat anti-rabbit IgG (H+L) (DyLight 633) (Invitrogen, 35562). 


\section{Transfections}

Primary astrocytes were transiently transfected with siRNA using Lipofectamine ${ }^{\mathrm{TM}}$ RNAiMAX Transfection Reagent (Invitrogen, 13778100) following manufacturer's instructions, with siRNA at $30 \mathrm{nM}$ final concentration. HeLa cells were transiently transfected with plasmdis using Lipofectamine ${ }^{T M} 2000$ Transfection Reagent (Invitrogen, 11668019) following manufacurere's instructions.

\section{Western blotting}

Cell lysates were retrieved using RIPA lysis buffer (Abcam, ab156034) supplemented with Protease Inhibitor Cocktail (Thermo Scientific, A32955) and PhosSTOP (Roche, 4906845001). Then cells were sonicated on ice and centrifuged at $4^{\circ} \mathrm{C}$ at $14000 \mathrm{~g}$ for $20 \mathrm{~min}$, followed by a BCA assay (Thermo Fisher Scientific, 23225) for protein concentrations. Next All cell lysates were boiled with $4 \times$ LDS sample buffer (Invitrogen, NP0007), 30-40 $\mu \mathrm{g}$ of total proteins were run out on a 4-12\% Sure PAGE Bis-Tris gel (Genscript, China, M00654) and transferred to PVDF membranes (Thermo Scientific, 88585). Membranes were probed with the indicated primary antibodies overnight at $4^{\circ} \mathrm{C}$, followed by the appropriate HRPconjugated secondary antibodies for $2 \mathrm{~h}$ at room temperature. The blots were imaged on the ChemiDoc developer system (Bio-Rad). All band detection was in the linear range. Related Information was provided in in Supplementary Figure 2.

\section{Co-immunoprecipitation (Co-IP)}

The cell lysates were extracted from the treated cells using cell lysis buffer (Cell Signaling Technology, 9803S) supplemented with Protease Inhibitor Cocktail (Thermo Scientific, A32955) and PhosSTOP (Roche, 4906845001), followed by a BCA assay (Thermo Fisher Scientific, 23225) for protein concentrations at $1 \mathrm{mg} / \mathrm{ml}$ and incubated with indicated primary antibodies overnight at $4^{\circ} \mathrm{C}$ with a constant shaking speed. Then, the complexes were mixed with Protein G Agarose (Roche, 11243233001) and shaken for $3 \mathrm{~h}$ at $4^{\circ} \mathrm{C}$ to capture the antigen-antibody mixture. The beads were then washed five times with cell lysis buffer and boiled in SDS loading buffer. The eluted proteins were analyzed by western blotting.

\section{Immunofluorescence}

Slide-cultured cells were treated as indicated in the figure legends. After treatment, the cells were fixed with 4\% paraformaldehyde at room temperature for $20 \mathrm{~min}$ and washed with PBS for $5 \mathrm{~min}$. Then, the cells were permeabilized with $0.1 \%$ Triton X-100 and blocked using $3 \%$ goat serum in PBS for 40 min. Next, the cells were incubated with primary antibodies (as indicated in the figure legends) diluted in $3 \%$ goat serum overnight at $4^{\circ} \mathrm{C}$, and then washed with PBS and incubated with anti-mouse Alexa-Fluor-488conjugated secondary antibodies and anti-rabbit Dylight-633-conjugated secondary antibodies for $1 \mathrm{~h}$ at room temperature. The cells were then washed thrice for 5 min each with $1 \%$ Triton X-100 in PBS. During the final wash step, they were incubated with DAPI (Solarbio, C0056) in PBS for 5 min. We used the Leica 
TCS SP5 confocal microscope and LSM 510 Zeiss confocal microscope for observing the immunofluorescence results.

\section{Flow cytometric analysis}

Cytokines in cultured cell supernatants were measured using a cytometric bead array (CBA) mouse Th1/Th2/Th1 Cytokine Kit (BD Biosciences, Cat\#560485), and IL-6, IL-10, TNF and IFN- $\gamma$ were selected as relative cytokines for astrocytes.

\section{Statistics}

Experiments were not randomized. All statistical data were calculated and graphed using GraphPad Prism6. All data are presented as means \pm SD. Statistical differences were detected using a two-tailed Student's t-test. A p-values less than 0.05 was considered statistically significant. ${ }^{*} p<0.05$, ${ }^{\star \star} p<0.01$, $\star \star \star p<0.001$.

\section{Results}

\section{The degradation of Cx43 is caused by autophagy after OGD}

Firstly, we found that the Cx43 protein levels decreased after OGD (Fig. 1a). We then wanted to confirm that this reduction was caused by autophagy. We first treated astrocytes with baf A1, an autophagy inhibitor, for 3 hours before and after OGD. Cx43 and P-Cx43 was found to have accumulated (Fig. 1b). Cx43 and P-Cx43 protein levels increased in Atg5 KD astrocytes after OGD for 6 hours (Fig. 1a). We also investigated the immunofluorescence of $\mathrm{Cx} 43$ and LC3B in primary astrocytes, which was found that overlay of two proteins increased after OGD(Fig. 1e). These results prove that the degradation of $\mathrm{Cx} 43$ is caused by autophagy after OGD.

OPTN and NDP52 are the autophagy receptors required for the autophagy of Cx43 in OGD

To ascertain whether OPTN and NDP52 were associated with the autophagy of Cx43, we chose siRNAs for their KDs. As shown in Fig. 2a, Cx43 and P-Cx43 protein levels were increased in OPTN KD astrocytes and NDP52 KD astrocytes after 6 hours OGD. To evaluate the relationship between Cx43 and OPTN and Cx43 and NDP52 at the spatial level, we used immunofluorescence analysis. The overlay of Cx43 and OPTN (Fig. 2C) and Cx43 and NDP52 (Fig. 2c) were found to increase in the primary astrocytes after 3 hours of OGD. Verifying the interaction between proteins requires Co-IP analysis. As shown in Fig. $5 \mathrm{a}$, the protein levels of OPTN and NDP52 that were combined with $\mathrm{Cx} 43$ increased after OA treatment, which means that the interactions between $\mathrm{C} \times 43$ and OPTN, as well as $\mathrm{C} \times 43$ and NDP52, had increased.

Also, we overexpressed RFP-OPTN and RFP-NDP52 in the HeLa cells. The interactions between Cx43 and RFP-OPTN, as well as Cx43 and RFP-NDP52, had increased after OGD (Fig. 3a), meanwhile, overlay of Cx43 and RFP also increased (Fig. 3b). Collectively, these findings suggest that OPTN and NDP52 are the autophagy receptors for the autophagy of Cx43 in OGD. 
PINK1 is the molecule involved upstream of the autophagy of Cx43 before the connections with NDP52 and OPTN in OGD

As shown in Fig. 4a, Cx43 protein levels were increased in PINK1 KD astrocytes after OGD, demonstrating that PINK1 was related to $\mathrm{Cx} 43$ degradation. We used Co-IP to detect binding between $\mathrm{C} \times 43$ and PINK1. We found that the PINK1 content bound to $\mathrm{Cx} 43$ had increased after OA treatment (Fig. 5a). Besides, overlay of Cx43 and PINK1 in primary astrocytes was found to had increased after OGD (Fig. 4C). These findings suggest PINK1 is associated with the autophagy of $\mathrm{C} \times 43$.

Next, we find that protein levels of OPTN and NDP52 that were combined with Cx43 disappeared after PINK1 KD in OGD (Fig. 5a). Meanwhile, the overlay between Cx43, RFP-OPTN and PINK1-Myc increased after OGD, the same as the overlay between Cx43, RFP-NDP52 and PINK1-Myc (Fig. 5b). All the above results prove that PINK1 is the molecule involved upstream of the autophagy of $\mathrm{Cx} 43$ before the connections with NDP52and OPTN under OGD.

The autophagy of $\mathrm{Cx} 43$ is related to ubiquitination in $\mathrm{OGD}$, which is composed of $\mathrm{pUb}(\mathrm{S} 65)$

Protein degradation requires ubiquitination, as does $\mathrm{Cx} 43$. The ubiquitin kinase PINK1 is closely related to the autophagy of $\mathrm{Cx} 43$. In this study, identify the ubiquitination used in the autophagy degradation of Cx43, we overexpressed HA-Ubquitin in Hela cells, which were then treated with OGD. We found that amounts of ubiquitin bound to $\mathrm{Cx} 43$ were increased (Fig. 6a),

As previously stated, the ubiquitin phosphorylation site was located at Ser65, which was phosphorylated by PINK1. Therefore, we next investigated the role of $\mathrm{pUb}(\mathrm{S} 65)$ in the autophagy of $\mathrm{Cx} 43$ in OGD. Co-IP showed that, after OGD, pUb(S65) modified Cx43 increased in HA-Ubquitin overexpressed Hela cells (Fig. 6a). Modification by pUb(S65) in the total protein content had increased in all groups after OGD (Fig. 6c). Altogether, these findings suggested that the ubiquitination of $\mathrm{Cx} 43$ consisted of pUb(S65) induced $\mathrm{Cx} 43$ autophagy.

The phosphorylation of Cx43 was involved in its autophagy

Changes in $\mathrm{Cx} 43$ ubiquitination revealed that some modifications to $\mathrm{C} \times 43$ must be occurring after OGD. Therefore, we chose phospho-Cx43(Ser368) as our target. First, pCx43(S368) protein levels were increased in Atg5KD, OPTN KD, NDP52KD and PINK1 KD astrocytes after OGD (Fig. 1a, Fig. 2a, Fig. 4a, and increased after baf $\mathrm{A} 1$ treatment (Fig. 1b). Next, Co-IP showed that RFP-OPTN and RFP-NDP52 that were bound to $\mathrm{pCx} 43$ (S368), had increased in HeLa cells after OGD (Fig. 3a). Then, amounts of ubiquitin bound to pCx43(S368) had increased in overexpressing HA-Ubiquitin Hela cells (Fig. 6b). As for pUb(S65), Co-IP showed that, after OGD, pUb(S65) modified pCx43(S368) had increased in overexpressed HAUbiquitin HeLa cells (Fig. 6b). Overall, the data showed that every change in pCx43(S368) was equal to that of $\mathrm{Cx} 43$, meaning that the phosphorylation of $\mathrm{Cx} 43$ was involved in its autophagy.

PKC and Src kinases are the start for Cx43 autophagy 
As mentioned above, Cx43 is phosphorylated by PKC and Src kinases at Ser368, Tyr265 and Tyr247 in OGD. We mutated the phosphorylation site of $\mathrm{Cx} 43$ to study the role of kinases. There is no doubt that NDP52, OPTN, PINK1, pUb(S65) bound to GFP-Cx43 had increased after OA treatment, but these connections decreased in the overexpressed GFP-Cx43(368A) Hela cells or overexpressed GFPCx43(247A + 265A) Hela cells (Fig. 7a). These findings suggest that PKC and Src kinases are the start of Cx43 autophagy.

Targeting Cx43 autophagy degradation pathway can improve neuroinflammation responses induced by OGD injury

We used flow cytometry with a CBA kit to measure concentrations of related cytokines (TNF, IL-10, IFN-y and IL-6) in cultured cell supernatants. TNF and IFN- $\gamma$ increased in WT astrocytes after OGD, while IL-10 and IL-6 decreased after OGD. Interestingly, TNF showed no significant difference in KD of ATG5, OPTN, NDP52 and PINK1 astrocytes after OGD, and IL-10 increased in these groups after OGD. IFN- $\gamma$ still increased and IL-6 decreased more (Fig. 8). These data suggest that blocking Cx43 autophagy degradation pathway can reduced pro-inflammatory cytokine concentrations (TNF and IL-6) and increased anti-inflammatory cytokine concentrations (IL-10) in OGD.

\section{Discussion}

Cx43 plays a vital role in maintaining the homeostasis of the nervous system. Previous studies have confirmed that $\mathrm{Cx} 43$ is dysfunctional under hypoxic conditions in the brain. $\mathrm{Cx} 43$ increases the release of excitatory glutamate, exacerbating the damage after ischemia ${ }^{[26,27]}$. Therefore, attention has been paid towards finding methods that can inhibit the function of $\mathrm{Cx} 43$, such as the addition of the $\mathrm{Cx} 43$ inhibitor $\mathrm{CBX}^{[28]}$ or the CX43-specific inhibitory peptide Gap $19^{[29,30]}$. However, only a small number of studies focus on changes in the amounts of $\mathrm{Cx} 43$ rather than channel permeability. Interestingly, some studies have reported that the degradation of $\mathrm{Cx} 43$ is achieved by selective autophagy under normal conditions. A related autophagy receptor that has been identified is $p 62^{[8]}$. Therefore, in this study, our hypothesis was that the brain has a targeting mechanism to clear $\mathrm{Cx} 43$ under ischemia conditions, which is selective autophagy.

Some previous research has shown that $\mathrm{C} \times 43$ increased after treatment with proteasomal inhibitors ${ }^{[31]}$, while other studies have described the genetic silencing of autophagy-associated genes resulted in accumulation of $\mathrm{Cx} 43$ and reduced co-localization of LC3B with $\mathrm{Cx} 43^{[32]}$. Simultaneously, decrease in Cx43 caused by starvation was sensitive to lysosomal inhibitors ${ }^{[32,33]}$. All above results predict both proteasomal pathway and autophagy can cause degradation of $\mathrm{Cx} 43$, but that the latter is more likely. Similarly, we demonstrated that the degradation of $\mathrm{Cx} 43$ under hypoxic conditions was achieved by autophagy. All these results predict that the degradation of Cx43 mainly depends on autophagy.

Since $\mathrm{C} \times 43$ degradation occurs via its autophagy under hypoxic conditions, the relevant autophagy receptors must play an important role. Previous literature has reported that OPTN and NDP52 are a pair 
of mitochondrial autophagy-related receptors ${ }^{[10]}$, while mitochondria contain $\mathrm{Cx} 43^{[11]}$, which is sensitive to ischemia. Therefore, the autophagy degradation of $\mathrm{Cx} 43$ may take a similar pathway. Unexpectedly, we show that NDP52 and OPTN are related to the autophagy receptors of Cx43 degradation. NDP52 and OPTN have UBDs as autophagy receptors ${ }^{[34]}$, which means the ubiquitination of $\mathrm{Cx} 43$ can happen before its connection with NDP52 and OPTN. The relationship between $\mathrm{C} \times 43$ degradation and ubiquitination has been reported in many studies. For example, E3 ubiquitin ligase Nedd4 interacts with the carboxyl terminus of $\mathrm{Cx} 43$ via its WW domains and ubiquitinates $\mathrm{Cx} 43^{[19,35]}$. Several E3 ubiquitin ligases are involved in Cx43 ubiquitination, including Wwp1 ${ }^{[36]}$, Smurf2 ${ }^{[37]}$, and TRIM21 $1^{[38]}$. Meanwhile, AMSHmediated deubiquitination of $\mathrm{C} \times 43$ regulates $\mathrm{C} \times 43$ degradation ${ }^{[39]}$. These studies showed that $\mathrm{C} \times 43$ ubiquitination was closely related to its degradation, and it was necessary to identify the ubiquitin kinase or ubiquitin ligase that involved. Recently, some studies have shown that the ubiquitin kinase PINK1 phosphorylates ubiquitin at Ser65 to activate mitophagy $[23,40]$, meaning that $\mathrm{pUb}(\mathrm{S} 65)$ may be involved in the degradation of $\mathrm{Cx} 43$. However, we are not clear about the relationship between $\mathrm{Cx} 43$ ubiquitination and its autophagy degradation in cerebral ischemia. We have shown that PINK1 is the ubiquitin kinase involved in this process and pUb(S65) modifies Cx43 in OGD injuries. In brief, Cx43 was phosphorylated in response to ischemia. Meanwhile, ubiquitin was phosphorylated by PINK1, and p-Cx43 was then modified with pS65-UB. Thus, this study confirmed that a modified form of ubiquitin, pS65-UB, modified Cx43.

Many studies have demonstrated that the phosphorylation of proteins affects their downstream autophagy. The phosphorylation of mAbg9 was shown to affect the molecular mechanism underlying its autophagic transport ${ }^{[41]}$. Another study showed that PTEN phosphorylation promotes the nuclear translocation and autophagy of PTEN in response to treatment with DNA-damaging agents in cancer cells $^{[42]}$. Phosphorylation is also related to the degradation of $\mathrm{Cx} 43^{[17]}$. Similarly, the phosphorylation of Cx43 may be associated with its autophagy. We chose the common Ser368, Tyr265 and Tyr247 sites of Cx43 according to previous studies ${ }^{[43,44]}$. After repeating the above experiments, it was found that the phosphorylation of $\mathrm{Cx} 43$ is closely related to its autophagy. When giving the above site a point mutation, we observed that the connection between cx43 and OPTN, NDP52 and PINK1 is weakened, simultaneously, the number of pS65-UB modified Cx43 also reduced. We show that phosphorylation of $\mathrm{Cx} 43$ is the starting point of its autophagy degradation.

IL-10, as an anti-inflammatory cytokine, has been shown to play an anti-inflammatory protective role in cerebral ischemia ${ }^{[45,46]}$, it enhances resistance to cerebral ischemia and reduces ischemic damage. Different from IL-10, TNF- $\mathbb{X}$ is a pro-inflammatory cytokine, as same as IL-6. A study showed that enhanced autophagy by rapamycin can upregulate IL-6 expression in mice astrocytes ${ }^{[47]}$. More interestingly, some links between $\mathrm{Cx} 43$ autophagy degradation and neuroinflammation were found in our work. Blocking $\mathrm{Cx} 43$ autophagy degradation pathway can reduced pro-inflammatory cytokine concentrations (TNF and IL-6) and increased anti-inflammatory cytokine concentrations (IL-10) in OGD. The above results reveal the relationship between autophagy degradation of Cx43 and astrocyte- 
mediated neuroinflammation. However, how autophagy degradation of $\mathrm{Cx} 43$ affects the production of

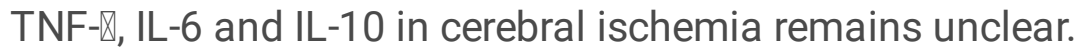

\section{Study Limitations}

This study has some limitations. Firstly, the phosphorylation site of $\mathrm{Cx} 43$ is not just the above three sites during OGD. Roles of MAPK and PKC in Cx43 autophagy degradation during OGD remains unknown. Furthermore, $\mathrm{Cx} 43$ autophagy degradation pathway in OGD is similar to mitophagy. The relationships between $\mathrm{Cx} 43$ and mitophagy need deeper research. Second, according to our results, Cx43 may be an anti-inflammation molecule. How $\mathrm{Cx} 43$ affects cytokines expression remains unclear. Vivo studies may provide more evidences.

\section{Conclusions}

In conclusion, we showed that under OGD, Cx43 is phosphorylated by PKC and Src kinases. Then phosphorylated $\mathrm{Cx} 43$ combines with pUb(S65) phosphorylated by PINK1. These modified $\mathrm{Cx} 43$ recruits the autophagy receptors OPTN and NDP52 for its autophagy-based degradation. Preventing the degradation of $\mathrm{Cx} 43$ autophagy is beneficial to the transformation of astrocytes to anti-inflammatory in cerebral ischemia. This study offers new ideas and intervention methods for Cx43-targeted therapy, bringing more crosstalk between autophagy and neuroinflammation.

\section{Abbreviations}

Cx43: connexin 43; baf A1: bafilomycin A1; OPTN: optineurin; pUb(S65): phosphorylated ubiquitin at Ser65; NDP52: calcium binding and coiled-coil domain 2; WT: wild type; KD: knock down; LC3: microtubule-associated protein 1 light chain 3; PINK1: PTEN induced putative kinase 1; PKC: protein kinase C; Atg5: autophagy-related 5; HA: hemagglutinin; GFP: green fluorescent protein; RFP: red fluorescent protein.

\section{References}

1.

Davidson JO, Green CR, Bennet L, et al. A key role for connexin hemichannels in spreading ischemic brain injury[J]. Curr Drug Targets. 2013;14(1):36-46.

2.

Montero TD, Orellana JA. Hemichannels: new pathways for gliotransmitter release[J]. Neuroscience. 2015;286:45-59.

3. 
Boengler K, Schulz R, Heusch G. Connexin 43 signalling and cardioprotection[J]. Heart. 2006;92(12):1724-7.

4.

Zeevi-Levin N, Barac YD, Reisner Y, et al. Gap junctional remodeling by hypoxia in cultured neonatal rat ventricular myocytes[J]. Cardiovasc Res. 2005;66(1):64-73.

5 .

Matsushita S, Kurihara H, Watanabe M, et al. Alterations of phosphorylation state of connexin 43 during hypoxia and reoxygenation are associated with cardiac function[J]. J Histochem Cytochem. 2006;54(3):343-53.

6.

Danon A, Zeevi-Levin N, Pinkovich DY, et al. Hypoxia causes connexin 43 internalization in neonatal rat ventricular myocytes[J]. Gen Physiol Biophys. 2010;29(3):222-33.

7.

Sato M, Jiao Q, Honda T, et al. Activator of G protein signaling 8 (AGS8) is required for hypoxia-induced apoptosis of cardiomyocytes: role of $\mathrm{G}$ betagamma and connexin 43 (CX43)[J]. J Biol Chem. 2009;284(45):31431-40.

8.

Fong JT, Kells RM, Gumpert AM, et al. Internalized gap junctions are degraded by autophagy[J]. Autophagy. 2012;8(5):794-811.

9.

Mizushima N, Komatsu M. Autophagy: renovation of cells and tissues[J]. Cell. 2011;147(4):728-41. 10.

Da ML, La S. K, et al. The ubiquitin kinase PINK1 recruits autophagy receptors to induce mitophagy[J]. Nature. 2015;524(7565):309-14.

11.

Azarashvili T, Baburina Y, Grachev D, et al. Calcium-induced permeability transition in rat brain mitochondria is promoted by carbenoxolone through targeting connexin43[J]. Am J Physiol Cell Physiol. 2011;300(3):C707-20.

12.

Solan JL, Lampe PD. Kinase programs spatiotemporally regulate gap junction assembly and disassembly: Effects on wound repair[J]. Semin Cell Dev Biol. 2016;50:40-8.

13.

Bright R, Raval AP, Dembner JM, et al. Protein kinase $\mathrm{C}$ delta mediates cerebral reperfusion injury in vivo[J]. J Neurosci. 2004;24(31):6880-8.

14.

Bai Y, Xu G, Xu M, et al. Inhibition of Src phosphorylation reduces damage to the blood-brain barrier following transient focal cerebral ischemia in rats[J]. Int J Mol Med. 2014;34(6):1473-82.

15.

Bright R, Mochly-Rosen D. The role of protein kinase $\mathrm{C}$ in cerebral ischemic and reperfusion injury[J]. Stroke. 2005;36(12):2781-90. 
16.

Liang S, Pong K, Gonzales C, et al. Neuroprotective profile of novel SRC kinase inhibitors in rodent models of cerebral ischemia[J]. J Pharmacol Exp Ther. 2009;331(3):827-35.

17.

Laird DW. Connexin phosphorylation as a regulatory event linked to gap junction internalization and degradation[J]. Biochim Biophys Acta. 2005;1711(2):172-82.

18.

Yin X, Liu J, Jiang JX. Lens fiber connexin turnover and caspase-3-mediated cleavage are regulated alternately by phosphorylation[J]. Cell Commun Adhes. 2008;15(1):1-11.

19.

Girao H, Catarino S, Pereira P. Eps15 interacts with ubiquitinated Cx43 and mediates its internalization[J]. Exp Cell Res. 2009;315(20):3587-97.

20.

Leithe E, Rivedal E. Epidermal growth factor regulates ubiquitination, internalization and proteasomedependent degradation of connexin43[J]. J Cell Sci. 2004;117(Pt 7):1211-20.

21.

Leithe E, Kjenseth A, Sirnes S, et al. Ubiquitylation of the gap junction protein connexin-43 signals its trafficking from early endosomes to lysosomes in a process mediated by Hrs and Tsg101[J]. J Cell Sci. 2009;122(Pt 21):3883-93.

22.

K FK. O, H K, et al. Ubiquitin is phosphorylated by PINK1 to activate parkin[J]. Nature. 2014;510(7503):162-6.

23.

Lazarou M, Sliter DA, Kane LA, et al. The ubiquitin kinase PINK1 recruits autophagy receptors to induce mitophagy[J]. Nature. 2015;524(7565):309-14.

24.

Yin X, Feng L, Ma D, et al. Roles of astrocytic connexin-43, hemichannels, and gap junctions in oxygenglucose deprivation/reperfusion injury induced neuroinflammation and the possible regulatory mechanisms of salvianolic acid B and carbenoxolone[J]. J Neuroinflammation. 2018;15(1):97. 25.

Ma D, Feng L, Cheng Y, et al. Astrocytic gap junction inhibition by carbenoxolone enhances the protective effects of ischemic preconditioning following cerebral ischemia[J]. J Neuroinflammation. 2018;15(1):198. 26.

Jc S, Vm B, Mc B, et al. Plasma membrane channels formed by connexins: their regulation and functions[J]. Physiological reviews. 2003;83(4):1359-400.

27.

Jc S, Ka S, Ma R, et al. Cell membrane permeabilization via connexin hemichannels in living and dying cells[J]. Experimental cell research. 2010;316(15):2377-89.

28. 
Hou S, Shen PP, Zhao MM, et al. Mechanism of Mitochondrial Connexin43's Protection of the Neurovascular Unit under Acute Cerebral Ischemia-Reperfusion Injury[J]. Int J Mol Sci, 2016, 17(5). 29.

Gadicherla AK, Wang N, Bulic M, et al. Mitochondrial Cx43 hemichannels contribute to mitochondrial calcium entry and cell death in the heart[J], 2017, 112(3): 27.

30.

Wang N, De Vuyst E, Ponsaerts R, et al. Selective inhibition of Cx43 hemichannels by Gap19 and its impact on myocardial ischemia/reperfusion injury[J]. Basic Res Cardiol. 2013;108(1):309. 31.

Musil LS, Le AC, Vanslyke JK, et al. Regulation of connexin degradation as a mechanism to increase gap junction assembly and function[J]. J Biol Chem. 2000;275(33):25207-15.

32.

Bejarano E, Girao H, Yuste A, et al. Autophagy modulates dynamics of connexins at the plasma membrane in a ubiquitin-dependent manner[J]. Mol Biol Cell. 2012;23(11):2156-69.

33.

Lichtenstein A, Minogue PJ, Beyer EC, et al. Autophagy: a pathway that contributes to connexin degradation[J]. J Cell Sci. 2011;124(Pt 6):910-20.

34.

Wild P, Mcewan DG, Dikic I. The LC3 interactome at a glance[J]. J Cell Sci. 2014;127(Pt 1):3-9. 35.

Leykauf K, Salek M, Bomke J, et al. Ubiquitin protein ligase Nedd4 binds to connexin43 by a phosphorylation-modulated process[J]. J Cell Sci. 2006;119(Pt 17):3634-42.

36.

Basheer WA, Harris BS, Mentrup HL, et al. Cardiomyocyte-specific overexpression of the ubiquitin ligase Wwp1 contributes to reduction in Connexin 43 and arrhythmogenesis[J]. J Mol Cell Cardiol. 2015;88:113.

37.

Fykerud TA, Kjenseth A, Schink KO, et al. Smad ubiquitination regulatory factor-2 controls gap junction intercellular communication by modulating endocytosis and degradation of connexin43[J]. J Cell Sci. 2012;125(Pt 17):3966-76.

38.

Chen VC, Kristensen AR, Foster LJ, et al. Association of connexin43 with E3 ubiquitin ligase TRIM21 reveals a mechanism for gap junction phosphodegron control[J]. J Proteome Res. 2012;11(12):6134-46. 39.

Ribeiro-Rodrigues TM, Catarino S, Marques C, et al. AMSH-mediated deubiquitination of Cx43 regulates internalization and degradation of gap junctions[J]. Faseb j. 2014;28(11):4629-41.

40.

Koyano F, Okatsu K, Kosako H, et al. Ubiquitin is phosphorylated by PINK1 to activate parkin[J]. Nature. 2014;510(7503):162-6.

41. 
Zhou C, Ma K, Gao R, et al. Regulation of mATG9 trafficking by Src- and ULK1-mediated phosphorylation in basal and starvation-induced autophagy[J]. Cell Res. 2017;27(2):184-201.

42.

Chen JH, Zhang P, Chen WD, et al. ATM-mediated PTEN phosphorylation promotes PTEN nuclear translocation and autophagy in response to DNA-damaging agents in cancer cells[J]. Autophagy. 2015;11(2):239-52.

43.

$\mathrm{Bf} R, \mathrm{Fm}$ JQ. V, et al. Phosphatase-resistant gap junctions inhibit pathological remodeling and prevent arrhythmias[J]. Circulation research. 2011;108(12):1459-66.

44.

Wayakanon P, Bhattacharjee R, Nakahama K, et al. The role of the Cx43 C-terminus in GJ plaque formation and internalization[J]. Biochem Biophys Res Commun. 2012;420(2):456-61.

45.

Liang QJ, Jiang $\mathrm{M}$, Wang $\mathrm{XH}$, et al. Pre-existing interleukin 10 in cerebral arteries attenuates subsequent brain injury caused by ischemia/reperfusion[J]. IUBMB Life. 2015;67(9):710-9.

46.

Tukhovskaya EA, Turovsky EA, Turovskaya MV, et al. Anti-inflammatory cytokine interleukin-10 increases resistance to brain ischemia through modulation of ischemia-induced intracellular $\mathrm{Ca}(2)(+)$ response[J]. Neurosci Lett. 2014;571:55-60.

47.

Zhang $\mathrm{Y}, \mathrm{He} \mathrm{X}$, Wu X, et al. Rapamycin upregulates glutamate transporter and IL-6 expression in astrocytes in a mouse model of Parkinson's disease[J]. Cell Death Dis. 2017;8(2):e2611.

\section{Declarations}

\section{Ethics approval and consent to participate}

The experimental protocols were approved by the Experimental Animal Research Ethics Committee of Jilin University.

\section{Consent for publication}

Not applicable

\section{Acknowledgements}

We would like to thank Editage (www.editage.cn) for English language editing.

\section{Funding}

This research was supported in part by the grants from National Key R\&D Program of China (2017YFC110304), the grants from the National Natural Science Foundation of China (No. 81771257), the grants from the National Natural Science Foundation for Young Scientists of China (No. 81701158), the grants from the National Natural Science Foundation for Young Scientists of China (No. 81801145), 
the grants from the Health Department of Jilin Province (No. 20180101161JC) and the grants from the Health Department for Young Scientists of Jilin Province (No. 20180520134JH).

\section{Author contributions}

Xinyu Wang contributed to the design of the research, executed immunoblotting, immunofluorescence, flow cytometry quantification, and analysis as well as preparing the draft of manuscript. Liangshu Feng contributed to the cellular protein collection with different agents and methods. Meiying Xin interpreted the data and contributed to the writing of the manuscript. Xu Wang and Pei Shang isolated and cultured primary astrocytes for further studies. Shuai Hou and Mingming Zhao were responsible for the drug application of the cultured cells. Yunhai Zhang and Yun Xiao contributed to the immunofluorescence. Yulei Hao contributed to the transfection. Jiachun Feng and Di Ma were responsible for the study design, applying for funding, and interpreting the data. All authors have read and approved the final version of the manuscript.

\section{Availability of data and materials}

The datasets used and/or analyzed during the current study are available from the corresponding author on reasonable request.

\section{Competing interests}

The authors declare that they have no competing interests.

\section{Figures}


a

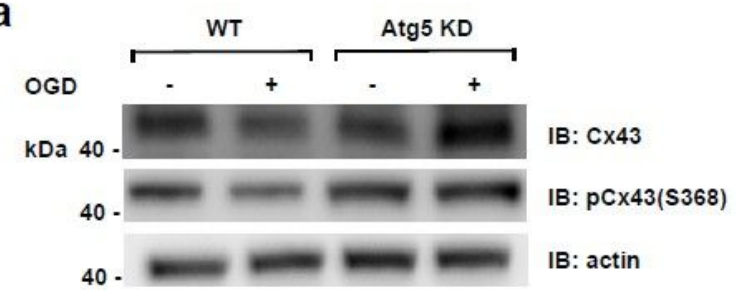

b

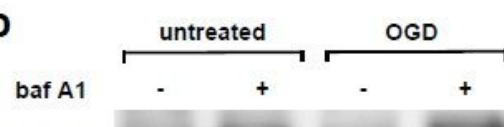

KDa 40 .

40 -

40 .

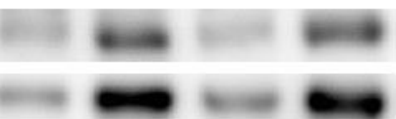

IB: Cx43

IB: pCx43(S368)

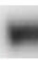

e

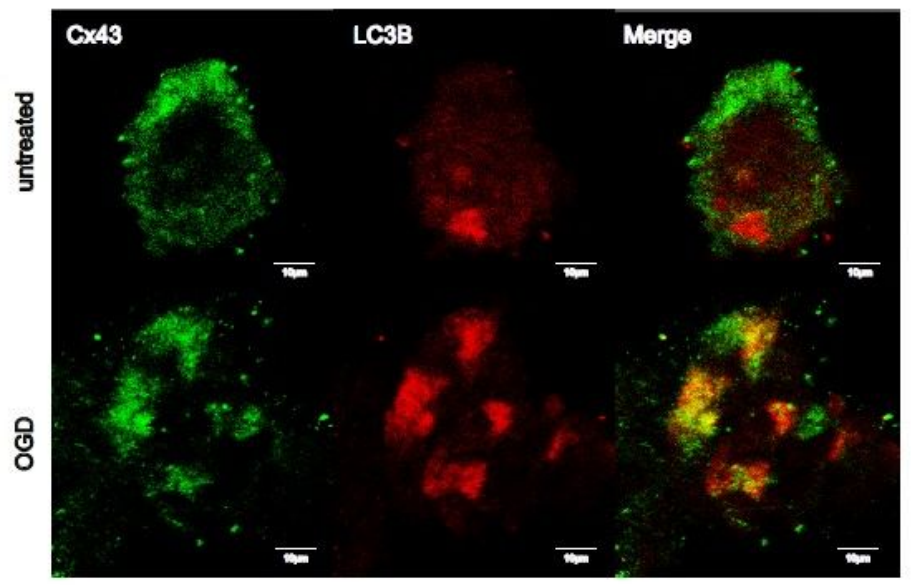

C

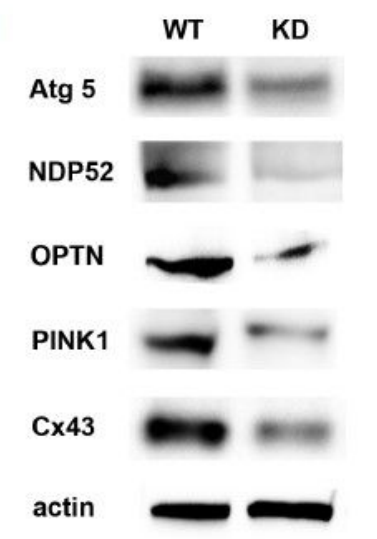

d

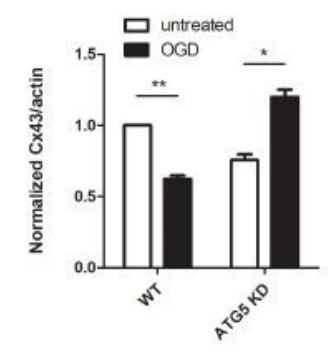

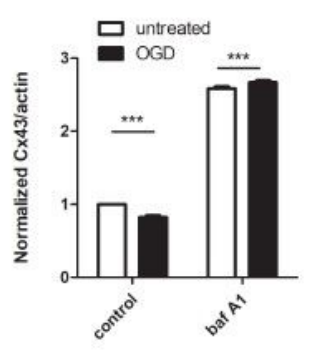

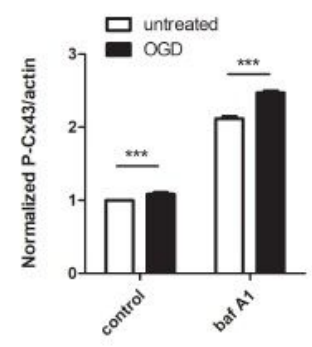

\section{Figure 1}

The degradation of $\mathrm{Cx} 43$ is caused by autophagy during OGD in primary astrocytes. a. Atg5 KD primary astrocytes and astrocytes transfected with negative control siRNA were treated with OGD for $6 \mathrm{~h}$ or left untreated. Representative protein bands are displayed. Western blot showed the increased levels of Cx43 and p-Cx43(Ser368) after Atg5 KD in OGD compared to negative control. b. Primary astrocytes with the addition of baf $A 1$ or not were treated with OGD for $6 \mathrm{~h}$ or left untreated. Representative protein bands are displayed. Western blot showed the increased levels of $\mathrm{Cx} 43$ and p-Cx43(Ser368) after the addition of baf A1. The graph reports means \pm SD of $\mathrm{Cx} 43$ /actin values from three independent experiments. $\mathrm{C}$.

Verification of gene knockdown. Primary astrocytes were transfected with negative control siRNA and related siRNAs. Representative protein bands are displayed. $d$. The graph reports means \pm SD of normalized $\mathrm{Cx} 43 /$ actin or $\mathrm{p}-\mathrm{C} \times 43(\mathrm{Ser} 368) /$ actin values from three independent experiments. $\mathrm{e}$. Representative fluorescent images demonstrated that overlay of Cx43 and LC3B increased in primary astrocytes after $3 \mathrm{~h}$ OGD. Scale bars, $10 \mu \mathrm{m}$. The error bar represented the mean $\pm S D(n=3) .{ }^{*} p<0.05$, ** $<0.01, \star \star \star p p<0.001$ by student t-test. 


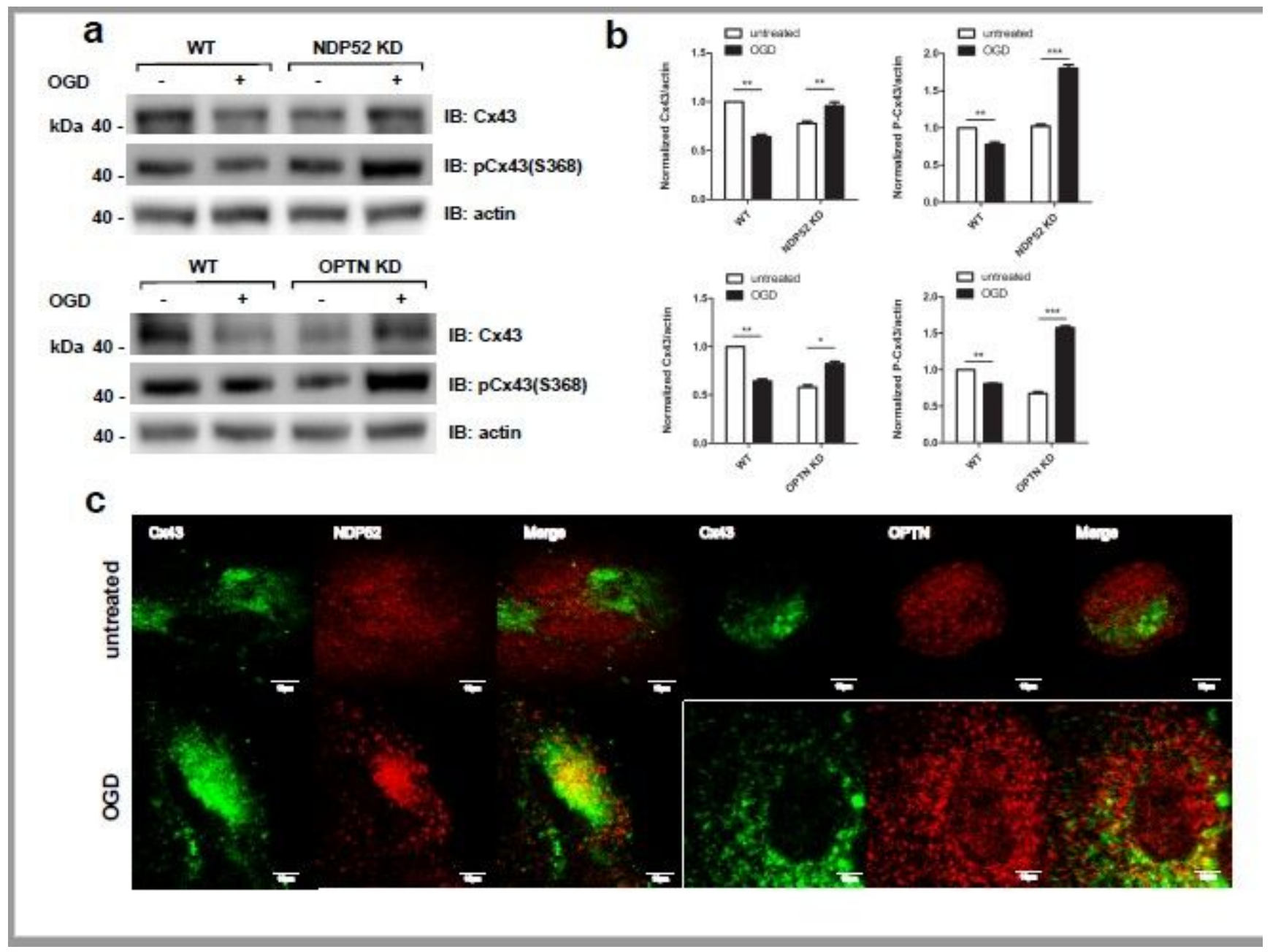

\section{Figure 2}

OPTN and NDP52 are receptors for the autophagy of Cx43 during OGD in primary astrocytes. a. NDP52 KD primary astrocytes and astrocytes transfected with negative control siRNA were treated with OGD for $6 \mathrm{~h}$ or left untreated. Western blot showed the increased levels of Cx43 and p-Cx43(Ser368) in NDP52 KD astrocytes after OGD. OPTN KD primary astrocytes and astrocytes transfected with negative control siRNA were treated with OA for $24 \mathrm{~h}$ or left untreated. Western blot showed the increased levels of $\mathrm{Cx} 43$ and p-Cx43(Ser368) in NDP52 KD astrocytes after OGD. b. The graph reports means \pm SD of normalized $\mathrm{Cx} 43 /$ actin or $\mathrm{p}-\mathrm{C} x 43$ (Ser368)/actin values from three independent experiments. c. Representative images of primary astrocytes showed overlay of Cx43 and NDP52, as well as Cx43 and OPTN, had increased with OGD for $3 \mathrm{~h}$. Scale bars, $10 \mu \mathrm{m}$. The error bar represented the mean $\pm S D(n=3) .{ }^{*} p<0.05$, ${ }^{\star *} \mathrm{p}<0.01,{ }^{* \star *} \mathrm{p}<0.001$ by student $\mathrm{t}$-test. 

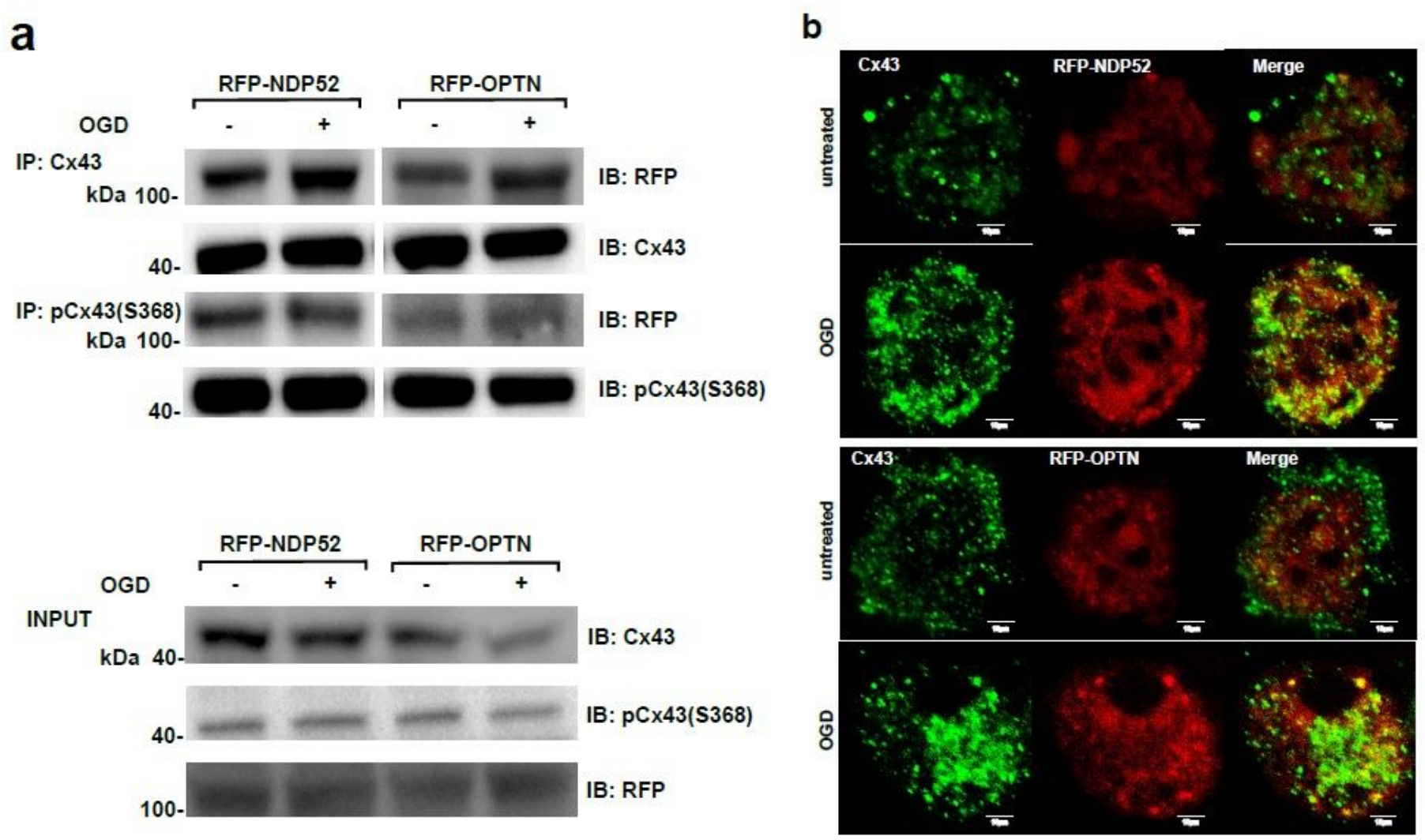

\section{Figure 3}

The interaction between Cx43 and RFP-NDP52 increased in HeLa cells during OGD, as well as Cx43 and RFP-OPTN. a. Protein extracts from RFP, RFP-OPTN- and RFP-NDP52-transfected Hela cells were subjected to immunoprecipitation using an anti-Cx43 and anti-pCx43(S368) antibody. Immunopurified complexes were analyzed by immunoblotting using anti-RFP antibodies. b. Fluorescent images of RFPOPTN- and RFP-NDP52-transfected Hela cells demonstrated that overlay of Cx43 and RFP-OPTN increased after 3h OGD, so did Cx43 and RFP-NDP52. Scale bars, $10 \mu \mathrm{m}$. 
a

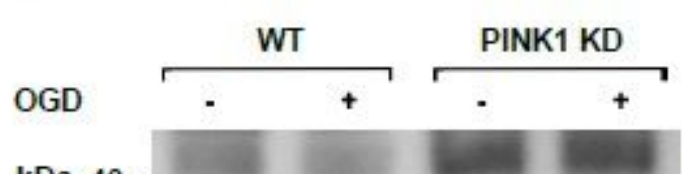

kDa 40 .

40

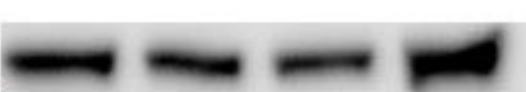

40 -

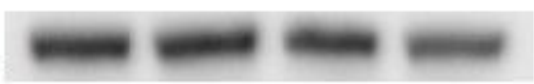

IB: actin

IB: $\mathrm{Cx} 43$ b

IB: pCx43(S368)
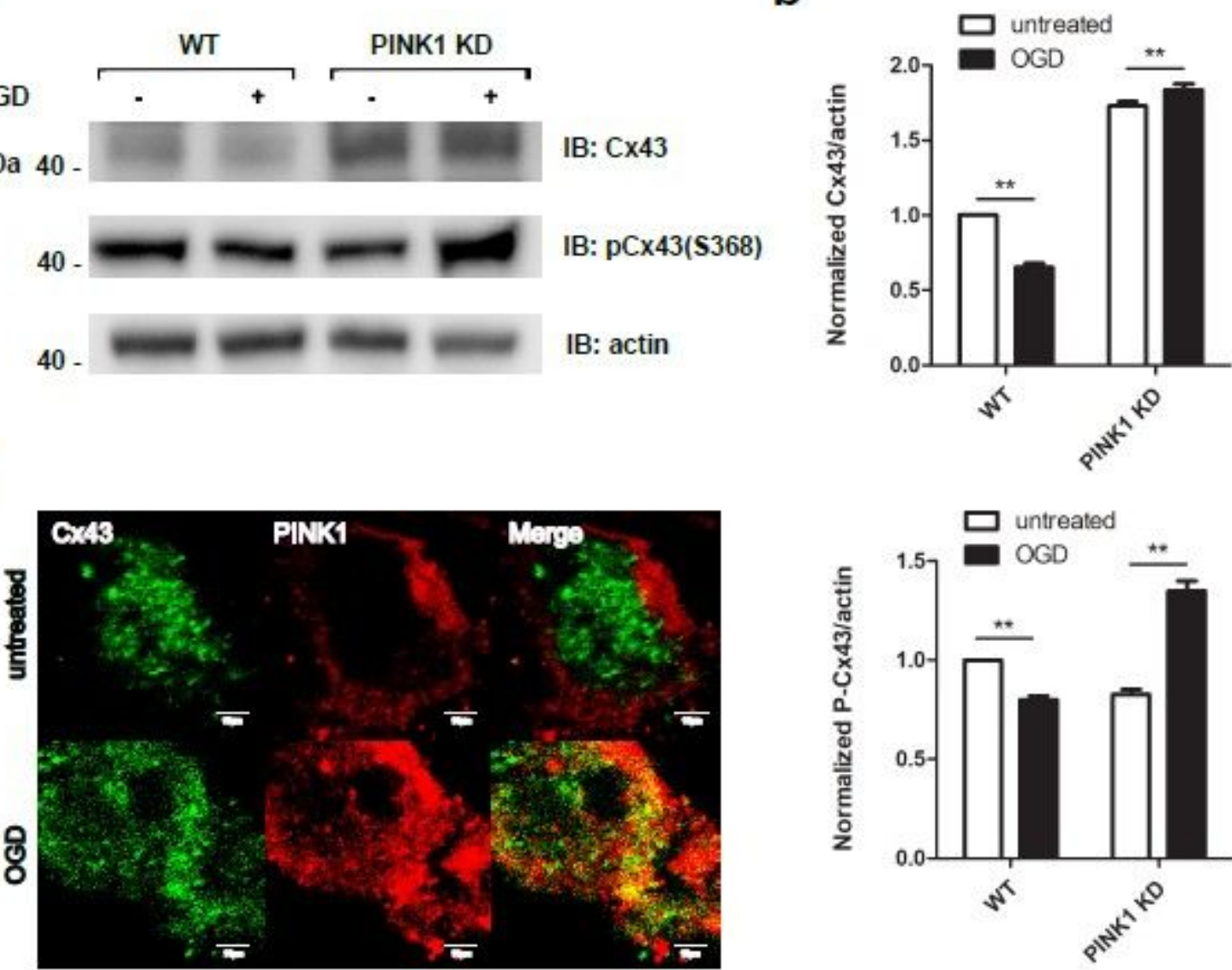

\section{Figure 4}

PINK1 is associated with $\mathrm{Cx} 43$ autophagy degradation during OGD. a. PINK1 KD primary astrocytes and astrocytes transfected with negative control siRNA were treated with OGD for $6 \mathrm{~h}$ or left untreated. Western blot showed the increased levels of Cx43 and pCx43(Ser368) in PINK1 KD astrocytes during OGD. b. The graph reports means \pm SD of normalized Cx43/actin or $\mathrm{p}-\mathrm{C} \times 43(\mathrm{Ser} 368) /$ actin values from three independent experiments. c. Representative fluorescent images demonstrated that overlay of $\mathrm{Cx} 43$ and PINK1 increased in astrocytes after 3h OGD. The error bar represented the mean $\pm S D(n=3)$. ${ }^{*} p<$ 0.01 by student $\mathrm{t}$-test. 
a
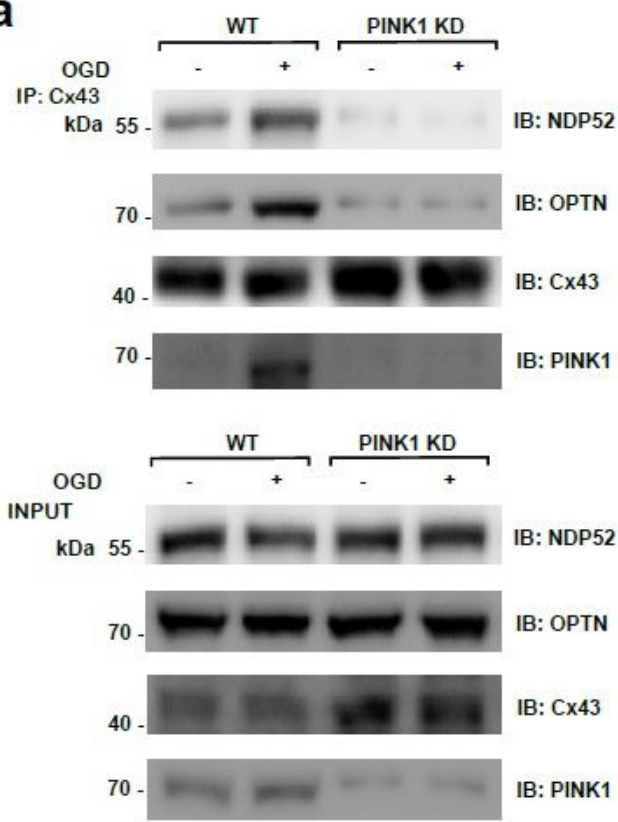

b
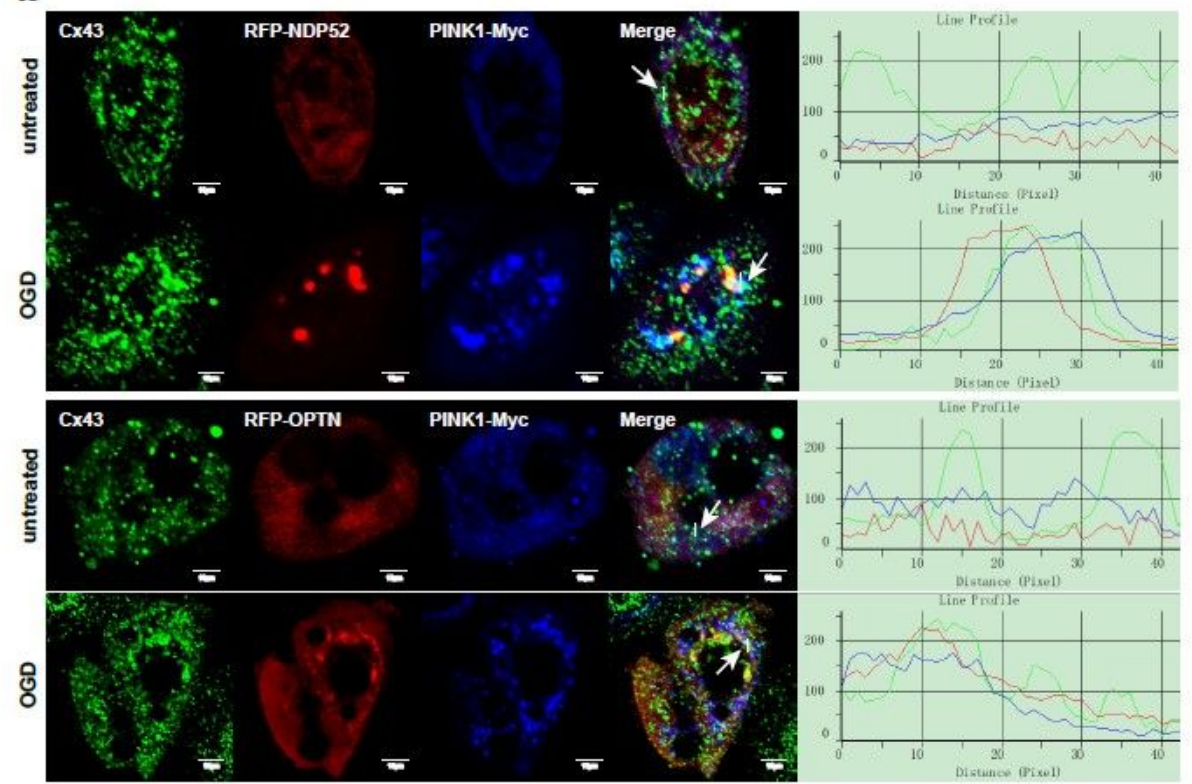

\section{Figure 5}

PINK1 is the molecule involved upstream of the autophagy of Cx43 in OGD. a. Protein extracts from PINK1 KD primary astrocytes and astrocytes transfected with negative control siRNA were subjected to immunoprecipitation using an anti-Cx43 antibody. Immunopurified complexes were analyzed by immunoblotting using anti-OPTN, anti-NDP52, anti-Cx43 and anti-PINK1 antibodies. OPTN and NDP52 that were combined with Cx43 increased during 3h OGD in primary astrocytes, while protein levels of OPTN and NDP52 that were combined with Cx43 disappeared after PINK1 KD in OGD. b. Fluorescent images of HeLa cells transfected both RFP-OPTN and PINK1-Myc or RFP-NDP52 and PINK1-Myc. The overlay between Cx43, RFP-OPTN and PINK1-Myc increased after OGD, the same as the overlay between Cx43, RFP-NDP52 and PINK1-Myc. Scale bars, $10 \mu \mathrm{m}$. 
a

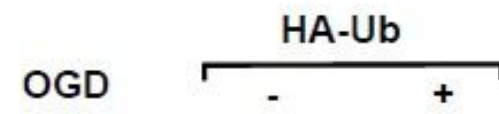

IP: Cx43

kDa 55-

55-

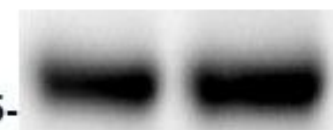

40-

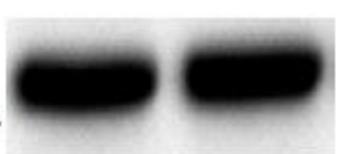

b

OGD

IP: pCx43(S368)

kDa 55-

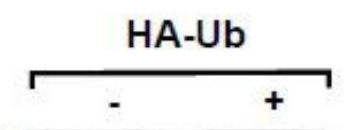

55-

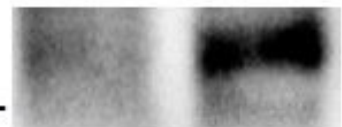

40-

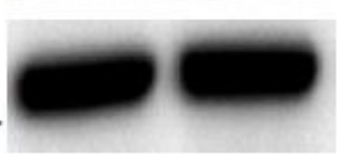

IB: HA

IB: pUb(S65)

IB: Cx43

IB: HA

IB: pUb(S65)

IB: $p C x 43(\$ 368)$

\section{C \\ INPUT \\ kDa \\ 40-}

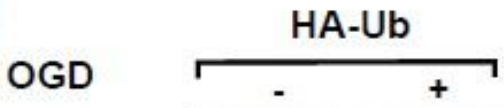

$40-$

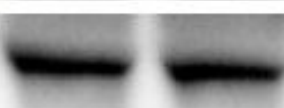

100 -

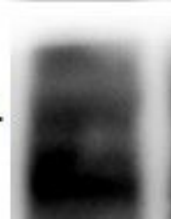

IB: $p C \times 43(\$ 368)$

IB: Cx43

IB: HA

$55-$

40.

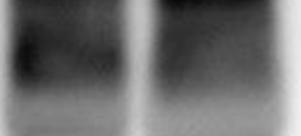

$100-$

IB: pUb(S65)

55-

40-

\section{Figure 6}

The autophagy of $\mathrm{Cx} 43$ depends on ubiquitination during OGD, which is composed of pUb(S65). a-b. Protein extracts from HA-Ub transfected Hela cells treated with 3h OGD or not were subjected to immunoprecipitation using an anti-Cx43 antibody or an anti-pCx43(S368) antibody. Immunopurified complexes were analyzed by immunoblotting using anti-HA and anti-pUb(S65) antibodies. Co-IP showed that $\mathrm{pUb}$ (S65) modified $\mathrm{Cx} 43$ remained same and $\mathrm{pUb}(\mathrm{S} 65)$ modified $\mathrm{p}-\mathrm{Cx} 43$ (Ser368) increased after $3 \mathrm{~h}$ OGD. c. Protein extracts from HA-Ub transfected Hela cells treated with 3h OGD or not were analyzed by immunoblotting using anti-Cx43, anti-pCx43(S368), anti-HA and anti-pUb(S65) antibodies. Ubiquitin- 
modified total protein remains essentially unchanged, while $\mathrm{pUb}(\mathrm{S} 65)$ modified total protein upregulated after OGD.

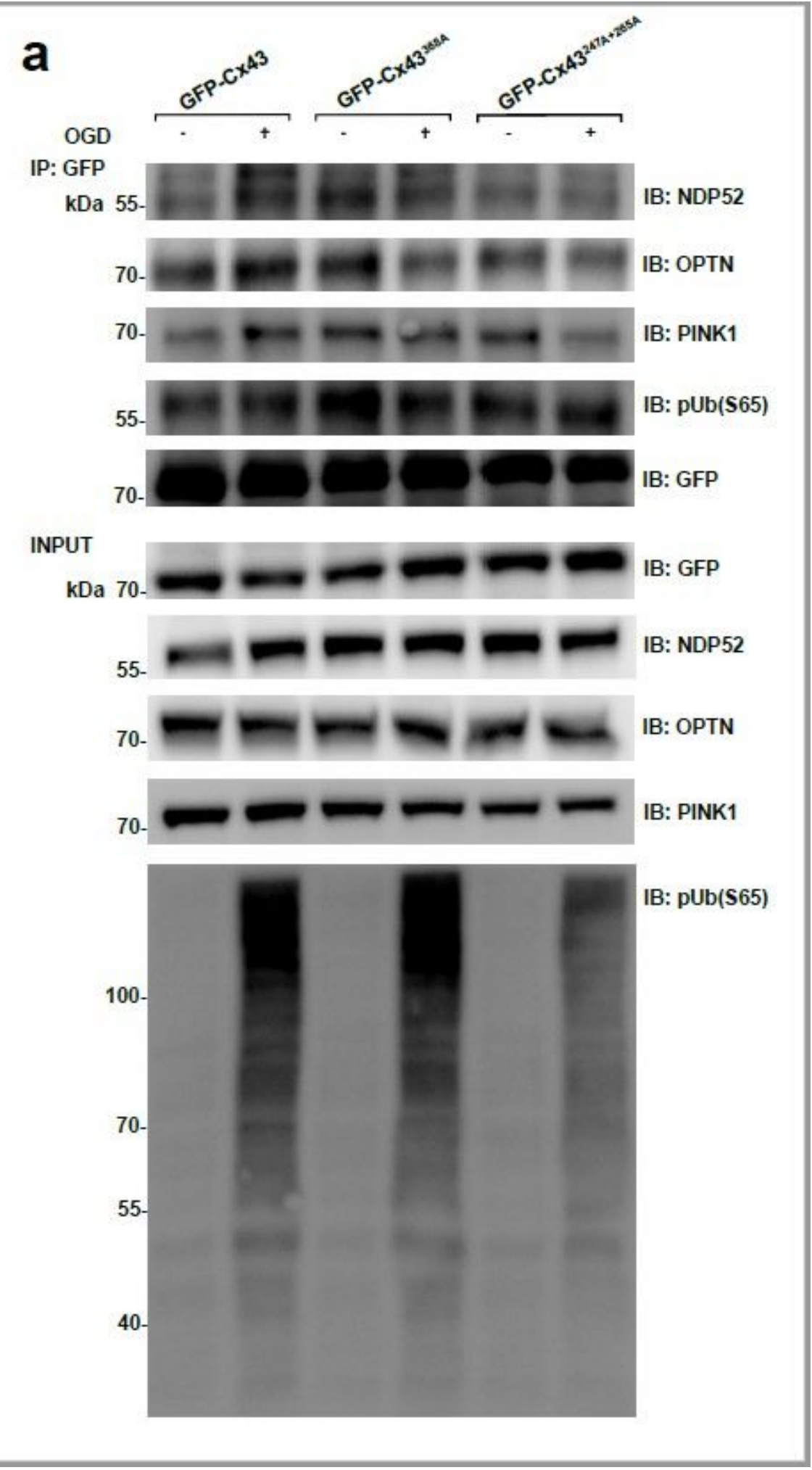

Figure 7

PKC and Src kinases are the start for Cx43 autophagy during OGD. a. Protein extracts from GFP-Cx43-, GFP-Cx43368A -and GFP-Cx43247A+265A -transfected Hela cells treated with 3h OGD or not were subjected to immunoprecipitation using an anti-GFP antibody. Immunopurified complexes were analyzed 
by immunoblotting using anti-OPTN, anti-NDP52, anti-PINK1 and anti-pUb(S65) antibodies. Co-IP showed that the connection between Cx43 and OPTN, NDP52 and PINK1 was weakened after point mutation and $\mathrm{pUb}(\mathrm{S} 65)$ modified Cx43 decreased. The INPUT protein extracts were also analyzed. The error bar represented the mean $\pm S D(n=4) .{ }^{*} p<0.05,{ }^{\star \star} p<0.01,{ }^{* \star} p<0.001$ by student $t$-test.
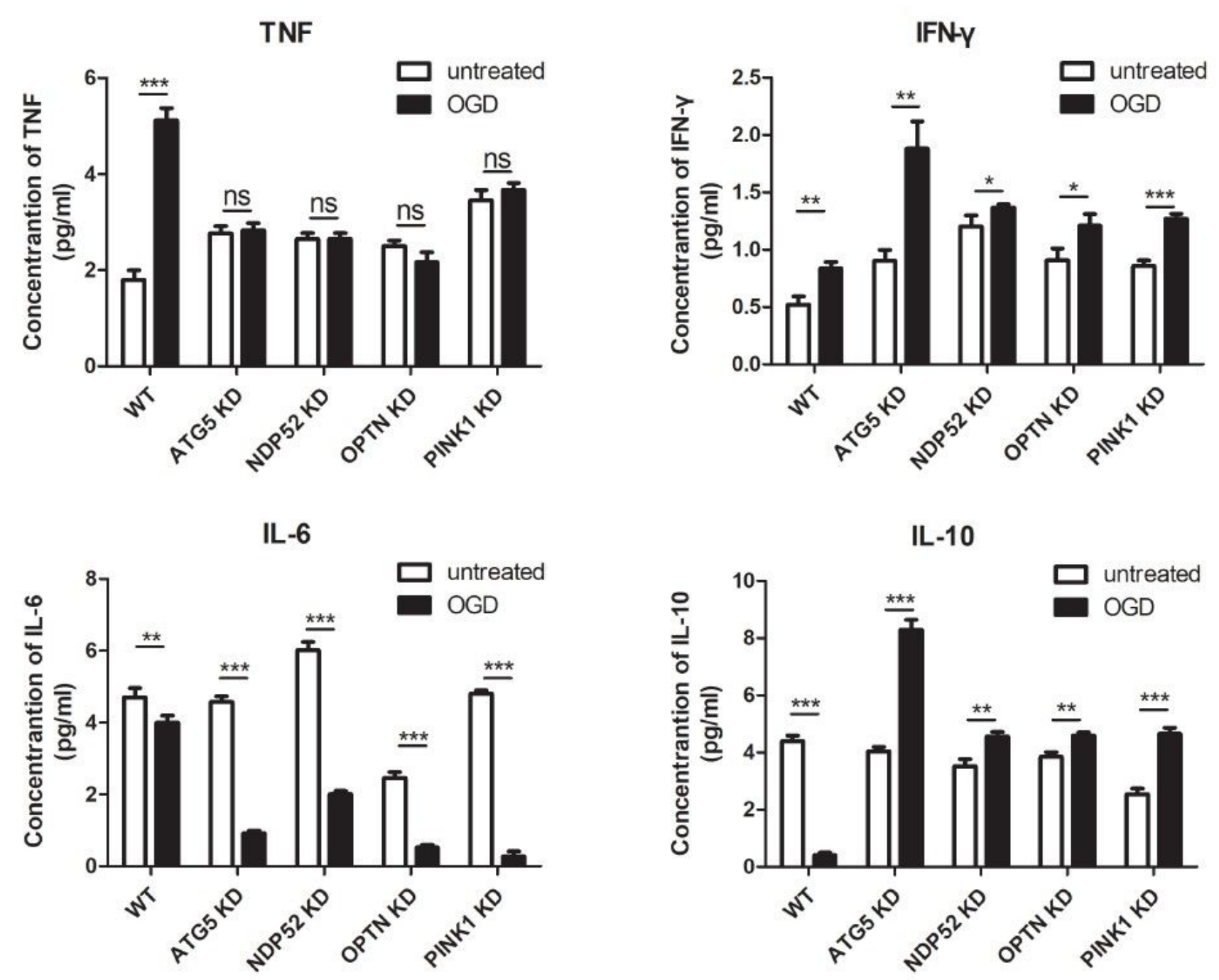

\section{Figure 8}

Flow cytometry-based evaluation of concentrations of cytokines in cultured astrocyte supernatants. cytokines were evaluated by flow cytometry with CBA kits. The error bar represented the mean $\pm S D(n=4)$. ${ }^{\star} \mathrm{p}<0.05,{ }^{\star \star} \mathrm{p}<0.01,{ }^{\star \star \star} \mathrm{p}<0.001$ by student t-test.

\section{Supplementary Files}

This is a list of supplementary files associated with this preprint. Click to download. 
- SupplementaryInformation.docx

Page 25/25 